\title{
The polycomb group protein EZH2 induces epithelial-mesenchymal transition and pluripotent phenotype of gastric cancer cells by binding to PTEN promoter
}

Lu Gan ${ }^{1,2,{ }^{\dagger}}$, Midie Xu ${ }^{4 \dagger}$, Ruixi Hua ${ }^{5 \dagger}$, Cong Tan ${ }^{6}$, Jieyun Zhang ${ }^{1,2}$, Yiwei Gong ${ }^{1,2}$, Zhenhua Wu ${ }^{1,2}$, Weiwei Weng ${ }^{6}$, Weiqi Sheng ${ }^{6}$ and Weijian Guo ${ }^{1,2^{*}}$

\begin{abstract}
Background: The influences of oncogenic Ezh2 on the progression and prognosis of gastric cancer (GC) and the underlying mechanisms are still poorly understood. Here, we aimed at investigating clinicopathological significance of Ezh2 in GC and the mechanisms underlying its function in GC development.

Methods: The expression level of Ezh2 was determined by qRT-PCR, immunoblot, and immunohistochemistry analysis in 156 pairs of GC tissues and adjacent normal gastric mucosa tissues. The biological functions of Ezh2 were assessed by in vitro and in vivo functional experiments. Chromatin immunoprecipitation (ChIP), luciferase, and Western blotting analyses were utilized to identify the relationship between Ezh2 and the PTEN/Akt signaling.

Results: The expression of Ezh2 was higher in gastric cancer tissues in comparison with para-nontumorous epithelium. High expression of Ezh2 was associated with more aggressive biological behavior and poor prognosis in GC. In vitro studies indicated that Ezh2 promoted GC cells' proliferation and clonogenicity. Besides, Ezh2 led to the acquisition of epithelial-mesenchymal transition (EMT) phenotype of GC cells and enhanced GC cell migration and invasion capacity. In particular, Ezh2 strengthened sphere-forming capacity of GC cells, indicating its role in the enrichment of GC stem cells. Furthermore, we found that PTEN/Akt signaling contributed to the effects of Ezh2 on cancer stem cells (CSC) and EMT phenotype in GC cells, and blocking PTEN signaling significantly rescued the effects of Ezh2.
\end{abstract}

Conclusions: Taken together, Ezh2 has a central role in regulating diverse aspects of the pathogenesis of GC in part by involving PTEN/Akt signaling, indicating that it could be an independent prognostic factor and potential therapeutic target.

Keywords: Ezh2, PTEN, Gastric cancer, Pluripotent phenotype, Epithelial-mesenchymal transition

\section{Background}

Gastric cancer (GC) is one of the highest incident cancers and the leading cause of cancer-related mortality worldwide [1]. In China, GC has become the third highest cause of cancer death, and the relative 5-year survival rate for GC is less than $30 \%$ [2, 3]. Adjuvant chemotherapy

\footnotetext{
* Correspondence: guoweijian1@outlook.com

${ }^{\dagger}$ Equal contributors

'Department of Medical Oncology, Fudan University Shanghai Cancer Center, Shanghai 200032, China

${ }^{2}$ Department of Oncology, Shanghai Medical College, Fudan University,

Shanghai 200032, China

Full list of author information is available at the end of the article
}

following a D2 resection is regarded as the standard curative treatments for localized GC, and the standard of care in the first line setting of metastatic disease remains a combination of fluoropyrimidine and platinum-containing chemotherapy [4]. However, the long-term prognosis of GC remains unsatisfactory because of the high rate of recurrence and metastasis. Thus, it is important to investigate the molecular mechanisms of GC progression and to identify genes implicated and the driver mutations/genetic alterations.

The enhancer of zeste homolog 2 (Ezh2) belongs to the family of polycomb group genes (PcGs). It has 
emerged as a master regulator of cell division [5], DNA repair [6], autophagy [7], signal transduction, and embryonic development [8]. Thus, Ezh2 has multiple essential biological effects, and it is not surprising that its function (and often dysfunction) plays an important role in various diseases, especially in cancer [9]. Increased Ezh2 expression has been reported in different types of cancers, including colorectal cancer [10], hepatocellular carcinomas [11], and lung cancer [12, 13]. Recently Ezh2 protein has been disclosed overexpressed and is associated with several tumor proliferation and invasion-associated genes, as well as the prognosis of GC [14-16]. A panel of genes including HOTAIR [17], CCAT2 [18], and TP53 [19] that implicated in the EMT or pluripotent phenotype have been reported to be acting as the upstream moleculars of Ezh2 dysregulation in GC. These findings raised the question that what the downstream of functional Ezh2 is in cancer EMT phenotype and sphere-forming capacity, resulting in the carcinogenesis and progression of GC.

Herein, we demonstrate that Ezh2 expression is correlated with poor survival in GC. Besides promoting cell proliferation and invasion, Ezh2 has a pivotal role in CSC enrichment and EMT of GC. Most importantly, we show that PTEN is a direct target of Ezh2. Ezh2 binds to the PTEN locus and downregulates PTEN expression, which consequently activates the Akt pathway, stabilizes Vimentin, downregulates E-cadherin, and protects Sox2 and Oct4 from degradation, thus ultimately leads to the acquisition of EMT and pluripotent phenotype of GC cells. Taken together, our results provided an explanation for the aggressive nature of human tumors overexpressing Ezh2 through a mechanism that links Ezh2 to the key tumor suppressor PTEN. These findings of the role of Ezh2 in the progression of GC may imply Ezh2 as a prognostic factor and potential therapeutic target.

\section{Methods}

\section{Human tissue specimens}

The human tissue specimens in this study were collected from 156 patients with histologically confirmed GC, who had underwent prior surgical resection and received no pretreatment. These samples were obtained from the tissue bank of Fudan University Shanghai Cancer Center (FUSCC) between 2008 and 2010. Independently, a total of 105 GC patient samples were enrolled for IHC analysis; all patients' formalin-fixed paraffin-embedded (FFPE) tissues were obtained from the department of pathology of FUSCC between 2005 and 2011. Follow-up was completed on November 30, 2016. The tumor grades were defined in accordance with the criteria outlined by the World Health Organization (WHO) Classification of Tumors of the Digestive System, 2010 edition [20]. The study complied with the regulations of the Ministry of Health of China and the WHO Research Ethics Review Committee international guidelines for research involving human subjects and the Declaration of Helsinki on the ethical principles for medical research involving human subjects. The survival analysis results of 599 GC tissue samples from GEO cohort (GSE14210, GSE15459, GSE22377, GSE29272, and GSE51105; $n=$ 593) are available at the KMPlot database (http:// kmplot.com).

\section{Chromatin immunoprecipitation assays}

Chromatin immunoprecipitation (ChIP) assays were performed as previously described [21]. Briefly, the cells were trypsinized and resuspended in lysis buffer, and nuclei were isolated and sonicated to shear the DNA to $500 \mathrm{bp}-1 \mathrm{~kb}$ fragments (verified by agarose gel electrophoresis). Equal aliquots of chromatin supernatants were subjected to overnight IP with different antibodies as indicated or anti-flag as a negative control. DNA was extracted and the PTEN promoter, as well as the first exon, was amplified by PCR. Sequences of the PCR primers are listed in Additional file 1: Table S6.

\section{Luciferase assays}

Cells were transfected with the pGL3-based constructs containing the PTEN promoter plus the Renilla luciferase plasmid (pRL-TK). Then, the cells were harvested after $48 \mathrm{~h}$ for firefly/Renilla luciferase assays using the Dual-Luciferase Reporter Assay System (Promega). Luciferase activities were normalized to the cotransfected pRL-TK plasmid (mean \pm SD).

Other methods used in this study were described in previous publications and are listed in the supplementary information [21-24].

\section{Reproducibility}

Each experiment was performed in triplicate, and the data are presented as the mean $\pm \mathrm{SD}$. The sphere formation assay, cell invasion assays, animal experiments, Western blot, and immunohistochemistry results are representative of three independent experiments.

\section{Statistical analysis}

All statistical analyses were performed using SPSS 22.0 (IBM, SPSS, Chicago, IL, USA) and GraphPad Prism version 6.0 (GraphPad Software, San Diego, CA, USA). Statistical tests for data between group analysis included the $X^{2}$-test, Student's two-tailed $t$ test, and one-way ANOVA. DFS (disease-free survival) and OS (overall survival) curves were calculated with the Kaplan-Meier method and were analyzed with the log-rank test. The DFS rate was calculated from the date of surgery to the date of progression (local and/or distal tumor recurrence) or to the date of death. The OS rate was defined as the 
length of time between the diagnosis and death or last follow-up. Univariate and multivariate analysis were fit using a Cox proportional hazards regression model. A threshold of $P<0.05$ was defined as statistically significant.

\section{Results}

\section{Ezh2 expression is correlated with the progression and} outcome of GC

To investigate the Ezh2 expression in GC progression, we first examined the expression of Ezh2 by realtime qRTPCR in 156 cases and immunoblot in randomly selected 20 cases. Ezh2 mRNA and protein expression was higher in GC tissues compared with the matched adjacent normal mucous (Fig. 1a, b). In addition, IHC was employed to examine the protein expression of Ezh2 in 105 cases of primary GC FFPE tissues. Immunoreactivity of Ezh2 was observed primarily in the cytoplasm. The positive expression of Ezh2 protein was observed in $72(68.6 \%)$ cases (Fig. 1c). These findings strongly indicated that Ezh2 is overexpressed in GC. We further analyzed the relationship between qRT-PCR and immunoblot result from the same 20 cases and found a positive correlation between EZH2's mRNA and protein expression (Fig. 1d).

Then, we analyzed the association between Ezh2 expression and clinicopathological parameters in both qRTPCR and IHC groups (Additional file 1: Table S1). Ezh2 mRNA expression levels in tumor tissues were categorized as low or high relative based on the median [25]. Statistical analyses revealed that Ezh2 mRNA expression strongly correlated with the tumor size $(P=0.003)$, lymphatic invasion $(P=0.019)$, and TNM stage $(P=0.016)$, indicating that Ezh2 overexpression is associated with the clinical progression of human GC. The results in the IHC cohort showed that Ezh2 protein expression was also correlated with the tumor size $(P=0.018)$, lymphatic invasion $(P=0.027)$ and TNM stage $(P=0.038)$.

To analyze the correlation between Ezh2 mRNA expression and prognosis of GC patients, disease-free survival (DFS) and OS curves were plotted according to Ezh2 expression level by Kaplan-Meier method and the log-rank test. Our result showed that positive Ezh2 protein expression also resulted in a significantly poorer OS in the IHC cohort ( $n=103, P=0.033$, Fig. 1e). Consistently, patients in FUSCC cohort $(n=156)$ with high Ezh2 mRNA expression had a significantly poorer DFS $(P=0.001$, Fig. 1f $)$ and OS $(P=0.033$, Fig. 1f $)$ than those with low Ezh2 expression. Moreover, high Ezh2 mRNA expression also resulted in a significantly poorer DFS $(n=$ 359, $P<0.001$; Fig. 1f) and OS $(n=593, P<0.001$; Fig. $1 f)$ in the GEO cohort (GSE14210, GSE15459, GSE22377, GSE29272, and GSE51105) available at the KMPlot database (http://kmplot.com). Univariate and multivariate Cox proportional hazards analyses showed that Ezh2 and tumor-node-metastasis (TNM) stage were independent prognostic factors for DFS and OS in patients with gastric cancer in the qRT-PCR cohort (Additional file 1: Table S2-3), while only TNM stage was the independent prognostic factor for OS in patients with gastric cancer in the IHC cohort (Additional file 1: Table S4).

\section{Ezh2 promotes GC cell proliferation and clonogenicity}

To further investigate the biological role of Ezh2 in GC, we measured the baseline levels of Ezh2 in five GC cell lines and compared with the normal human gastric mucous cell line (GES-1). The Ezh2 level was significantly elevated in GC cells compared with that in the GES-1 cell (all $P<0.01$, Fig. 2a). Based on the baseline levels of Ezh2, the MKN-45 and SGC-7901 GC cell lines were selected for Ezh2 overexpression, and the AGS cell line was selected for knockdown of Ezh2. The efficiencies of overexpression and knockdown were confirmed by Western blotting $(P<0.01$, Fig. 2a). We found that Ezh2-overexpressing cells showed a significantly higher in vitro proliferation rate than control cells $(P<0.01$, Fig. 2b), while knockdown of Ezh2 suppressed cell proliferation in AGS cells in the CCK- 8 assays $(P<0.01$, Fig. $2 \mathrm{~b})$ and colony-forming assays $(P<0.01$, Fig. 2 c). Moreover, the mouse xenograft models showed that Ezh2knockdown cells (AGS-shEzh2) also generated smaller xenografts in nude mice than the control. In accordance with the reported role of Ezh2 in promoting growth, our results indicate that Ezh2 stimulated tumor growth (Fig. 2d).

\section{Ezh2 induces GC cell sphere-forming capacity and EMT phenotype}

Our clinical analysis found that Ezh2 overexpression was significantly correlated with GC tumor lymphatic invasion. We next asked if Ezh2 would affect GC cell invasion. As hypothesized, by the transwell assay, Ezh2overexpressing cells exhibited a significant increase of invasion, whereas Ezh2 knockdown showed significantly decreased invasion $(P<0.01$, Fig. 3a).

EMT was a key initial step during tumor invasion. For the detection of the correlation between EZH2- and EMT-related proteins in human gastric cancer, we did mRNA sequencing in EZH2 knockdown cells and then analyzed the gene set enrichment for EMT, which showed a good evidence for EZH2's regulation on EMT at the molecular level (Fig. 3c). Further validation showed that Ezh2-overexpressing cells exhibited a significant decrease in expression of the epithelial marker E-cadherin and increase in expression of the mesenchymal marker Vimentin, while silencing of Ezh2 expression in AGS cells resulted in increased E-cadherin expression and decreased Vimentin expression (Fig. 3d). These findings demonstrate that Ezh2 could regulate the phenotypic shift of EMT/ MET and promote cell invasion. Consistently, immunohistochemical staining demonstrated higher expression of E- 

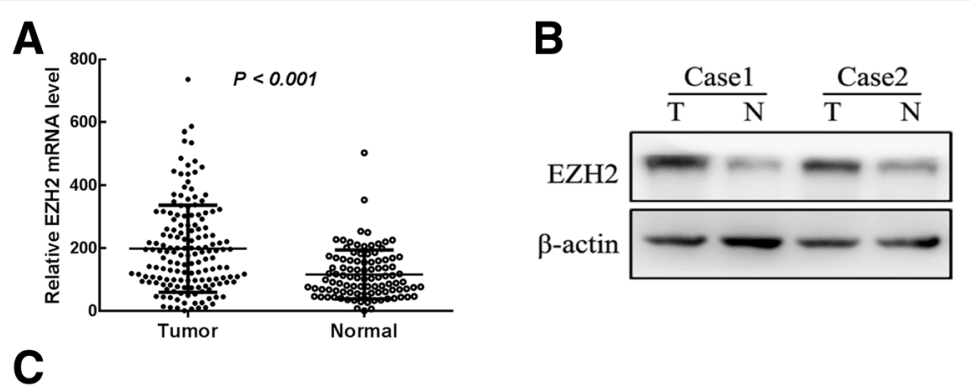

C

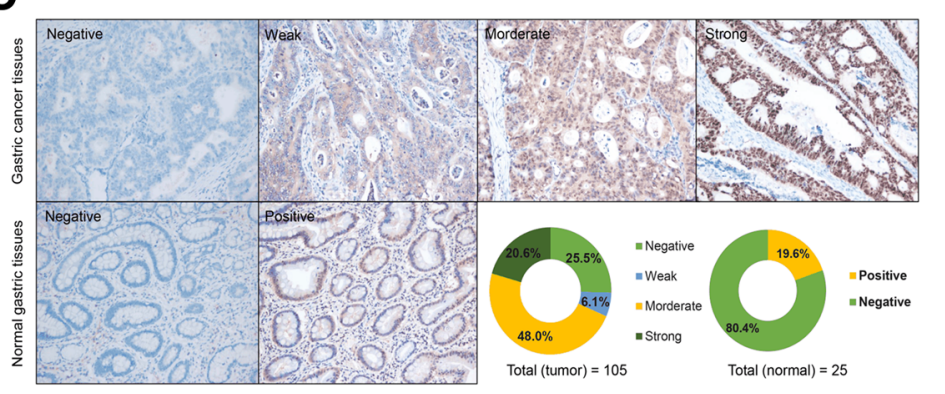

D
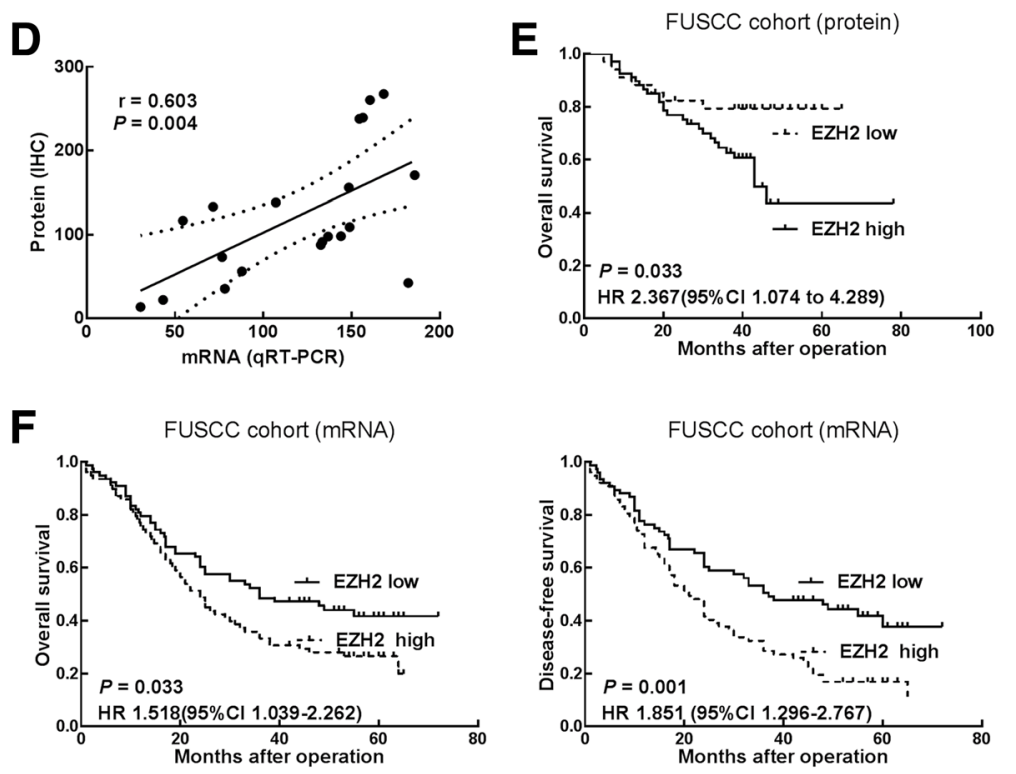

G
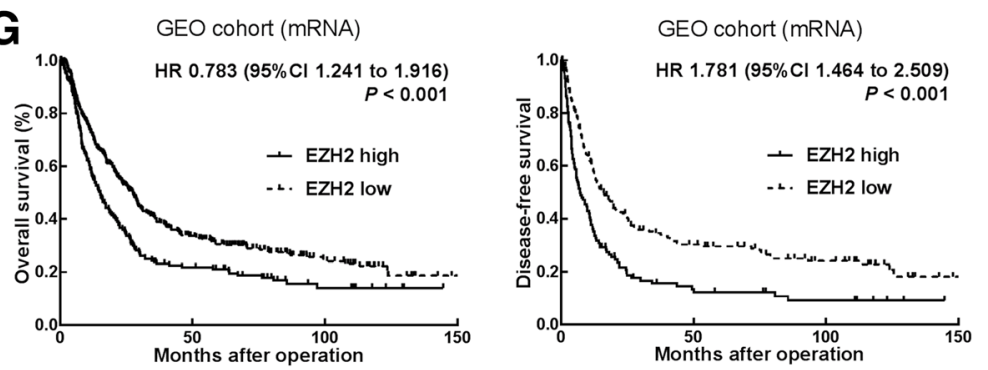

Fig. 1 (See legend on next page.) 
(See figure on previous page.)

Fig. 1 Ezh2 is upregulated in human GC tissues and associated with poor prognosis of GC. a The qRT-PCR results showed that the levels of Ezh2 mRNA in 156 pairs of GC tissues were significantly higher than those in adjacent normal mucosa. Data are represented as mean \pm SD. $\mathbf{b}$ Representative band of Western blotting showed that Ezh2 protein expression in GC tissues was higher than those in adjacent normal mucosa. c Representative image and the percentage of gastric cancer or normal gastric tissues with low or high Ezh2 protein expression. $\mathbf{d}$ Correlation analysis showed a positive correlation between EZH2's mRNA and protein expression in the same 20 cases. Data were analyzed by Spearman's correlation. e Kaplan-Meier survival curves with a log-rank test in the IHC group showed that the OS $(n=105)$ rates of the high EZH2 expression group were significantly lower than that of the low expression group $(P=0.033)$. The HRs and $P$ values were calculated with log-rank tests. $\mathbf{f}$ Kaplan-Meier survival curves showed poor disease-free survival (DFS) and overall survival in patients (FUSCC cohort, $n=156$ ) with high expression of Ezh2 compared with those in the low expression group. HRs and $P$ values were calculated with log-rank tests. $\mathbf{g}$ Kaplan-Meier survival curves showed poor disease-free survival (DFS, $n=359)$ and overall survival $(n=593)$ in patients (GEO cohort) with high expression of Ezh2 compared with those in the low expression group. HRs and $P$ values were calculated with log-rank tests
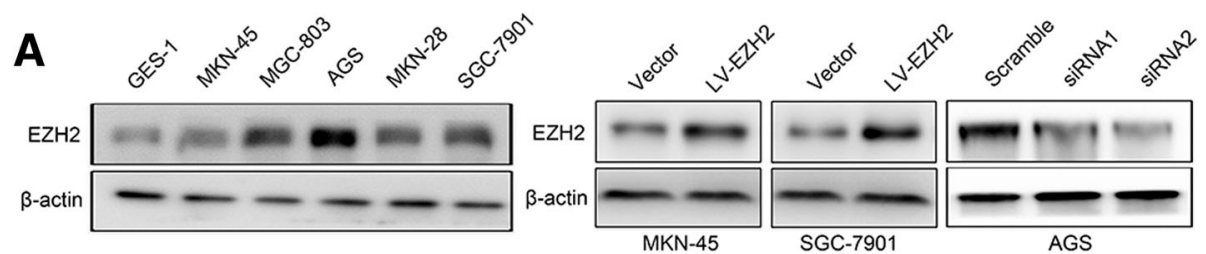

\section{B}
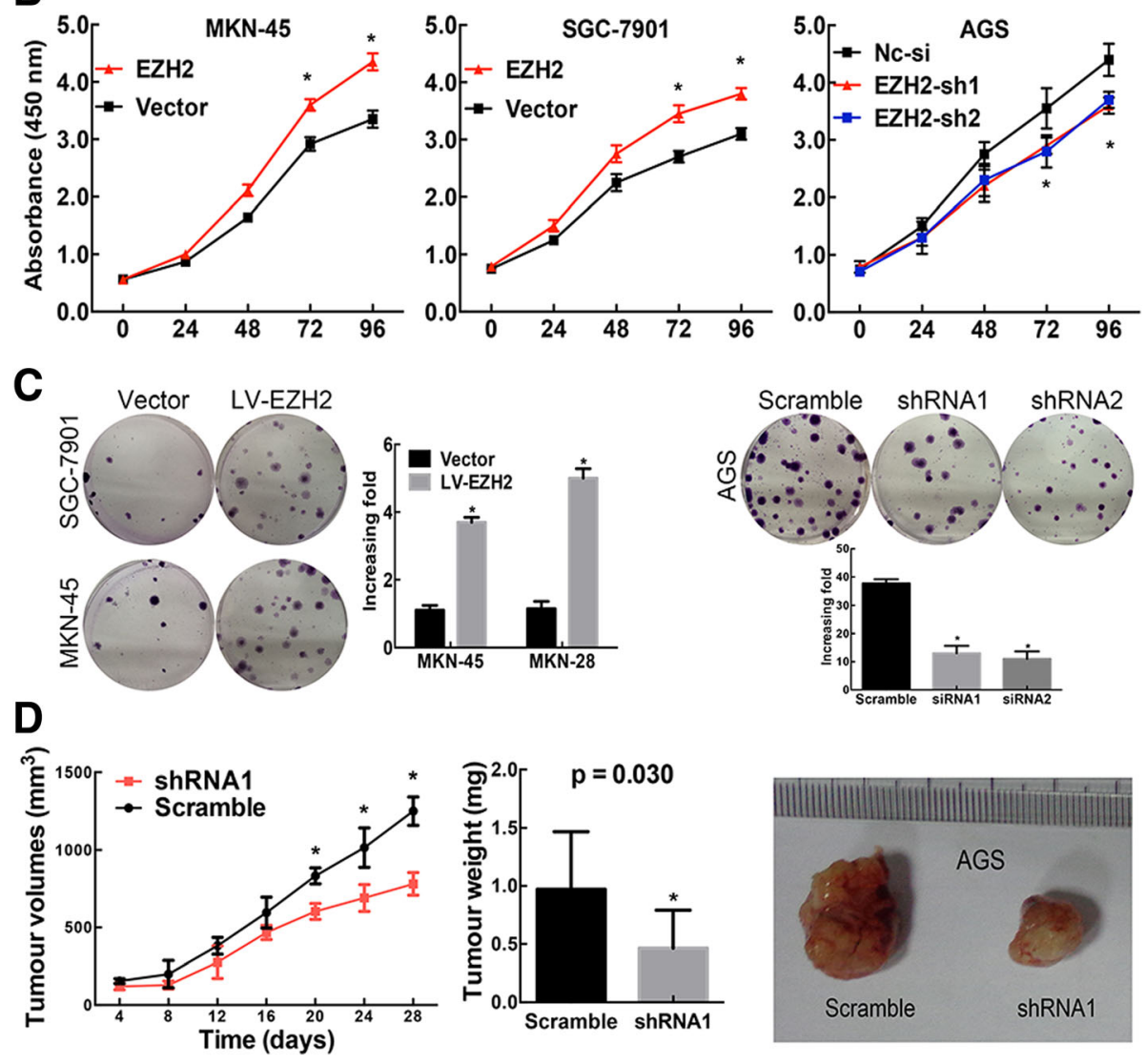

Fig. 2 Ezh2 promotes GC cell proliferation, clonogenicity, invasiveness and sphere-forming capacity. a The Western blotting results show the baseline protein levels of Ezh2 in GES-1 cell line and five GC cell lines and the efficiencies of Ezh2 overexpression in MKN-45 and SGC-7901 cells and Ezh2 knockdown in AGS cells. ${ }^{*} P<0.01$. $\mathbf{b}$ The CCK-8 assays showed that overexpression of Ezh2 promoted cell proliferation in MKN-45 and SGC-7901 cells, while knockdown of Ezh2 suppressed cell proliferation in AGS cells. Data are represented as mean \pm SEM. * $P<0.01$. c The colony-forming assays showed that overexpression of Ezh2 promoted cell colony-forming in MKN-45 and SGC-7901 cells, while knockdown of Ezh2 suppressed cell colony-forming in AGS cells. Data are represented as mean \pm SEM. ${ }^{*} P<0.01$. d Ezh2-knockdown AGS or scrambled-transfected AGS cells were injected into nude mice $(n=5)$ subcutaneously $\left(3 \times 10^{6}\right.$ per mouse). The nude mouse xenograft model showed that knockdown of Ezh2 decreased tumor growth (d left) and reduced tumor weights (d middle) compared with the scramble groups. Data are represented as mean \pm SEM. ${ }^{*} P<0.01$. The representative images of tumors were graphed (d right) 

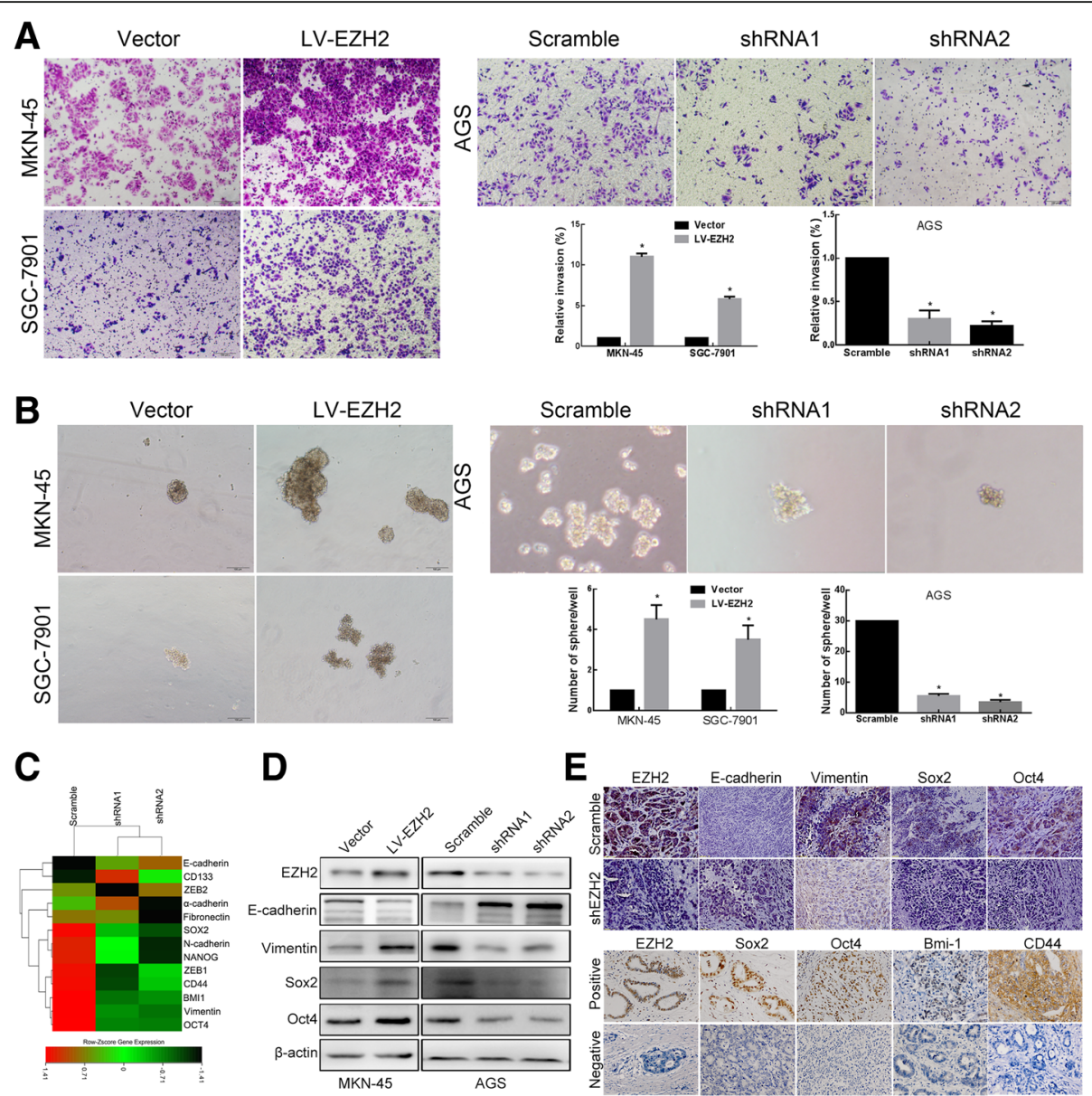

Fig. 3 Ezh2 regulates the expression of EMT, pluripotent protein, and PTEN/AKT signaling in GC. a Representative images and the number of invasive cells per high-power field (100X) showed that cell invasiveness was promoted by overexpression of Ezh2 in MKN-45 and SGC-7901 cells but suppressed by Ezh2 knockdown in AGS cells. Data are represented as mean $\pm S E M$. ${ }^{*} P<0.01$. b Representative images and the number of sphere formation per high-power field (100x) showed that stem cell-like properties were promoted by overexpression of Ezh2 in MKN-45 and SGC-7901 cells but suppressed by Ezh2 knockdown in AGS cells. Data are represented as mean \pm SEM. * $P<0.01$. c Heat-map display showed that the Ezh2-altered genes involved in EMT and cell stemness a. Gene expression profiling was performed with mRNA sequencing. $\mathbf{d}$ Representative images of the Western blot analysis for expression of Ezh2, E-cadherin, Vimentin, Oct4, and Sox2 in Ezh2-overexpressing MKN-45 cells and normal control, as well as Ezh2-knockdown AGS cells and normal control. e Representative images of the IHC analysis for expression of Ezh2, E-cadherin, Vimentin, Oct4, and Sox2 in xenograft tissues (up) and expression of Ezh2, Oct4, Sox2, Bmi-1, and CD44 in human GC and normal gastric mucous (down)

cadherin and lower expression of Vimentinin in Ezh2knockdown tissues of xenograft tumors (Fig. 3e), suggesting that knockdown of Ezh2 attenuates the EMT in vivo.

We also studied the effect of Ezh2 on the stem cell-like phenotype by sphere formation assays. The results showed that Ezh2-overexpressing cells exhibited a significant increase of sphere-forming efficiency, whereas Ezh2 knockdown cells showed significantly decrease sphereforming efficiency $(P<0.01$; Fig. 3b). Similarly, mRNA sequencing in EZH2 knockdown cells showed a good evidence for EZH2's regulation on cell stemness at the molecular level (Fig. 3c), and further validation showed that protein expression levels of the pluripotent genes, Oct4 and Sox2, were both significantly upregulated in Ezh2-overexpressing cells, whereas significantly reduced in Ezh2-knockdown GC cells compared with the control group $(P<0.01$, Fig. $3 \mathrm{~d})$. Consistently, immunohistochemical staining demonstrated higher expression of Oct4 and Sox2 in Ezh2-overexpressing tissues in xenograft tumors (Fig. 3e up). And our IHC results also showed a significant relationship between EZH2 protein and multiple pluripotent phenotype-related proteins (OCT4, BMI1, CD44; all $P<0.05$ ) in human GC tissues (Fig. 3e down, Additional file 1: Table S5). All these suggested that Ezh2 enhances the stem cell-like phenotype of GC cells.

\section{Ezh2 regulates PTEN/AKT signaling by directly binding to the promoter regions of PTEN in GC}

It has been reported that the PTEN/AKT signaling is a pivotal signaling pathway involved in EMT; PTEN 
accumulation in tumor cells might negatively regulate Akt by decreasing Akt phosphorylation level, thus contributes to E-cadherin expression increase and suppression of the EMT [26, 27]. In addition, PTEN and AKT signaling are both implicated in the regulation of cancer stem cell-like phenotype [28, 29]. However, whether Ezh2 promotes tumor cell EMT and enhances the stem cell-like phenotype via AKT/PTEN signaling pathway in gastric cancer warrants validation.

In the present study, we found that the Akt phosphorylation and PTEN expression varied with Ezh2 expression, PTEN was significantly decreased while phosphorylated Akt was significantly elevated in Ezh2-overexpressing cells, whereas both expressions were reversed in Ezh2-knockdown cells (Fig. 4a). We then detected the expression of PTEN in different gastric cell lines and find a negative correlation with Ezh2 (Fig. 4b). Consistently, immunohistochemical staining demonstrated lower expression of PTEN and higher level of phosphorylated Akt in Ezh2knockdown xenograft tumors (Fig. 4c), suggesting that Ezh2 may modulate the AKT/PTEN signaling. Moreover, the expression data in gastric cancer tissue samples from TCGA database also reveal a significant negative correlation between Ezh2 and PTEN mRNA in human gastric cancer samples (Fig. 4d).

Considering that Ezh2 is a Polycomb-group (PcG) family member that fulfill its oncogenic functions by participating in maintaining the transcriptional repressive state of genes over successive cell generations, we proposed that Ezh2 may be a transcriptional regulator of PTEN. Then, we determined whether Ezh2 regulates PTEN at the transcriptional level, our qRT-PCR results showed that aberrant expression of Ezh2 indeed affected PTEN mRNA expression; the mRNA levels of PTEN were reduced in Ezh2overexpressing MKN-45 and MKN-28 cells, while they were increased in Ezh2-knockdown AGS cells (Fig. 4e). We further investigated the regulatory mechanism underlying the correlation between Ezh2 and PTEN by dual-luciferase reporter assays, our results indicated that overexpression of Ezh2 reduced the PTEN promoter activity in HEK-293T and MKN45 cells (Fig. 4f). ChIP assays were performed to investigate whether Ezh2 associates with the PTEN locus. We proposed that Ezh2 binds to the region of the Pten promoter from -0.9 to $-2.3 \mathrm{~kb}$ and from 0.4 to $1.4 \mathrm{~kb}$; for determination of the exactly binding region, we allocate the Pten promoter into 10 sequences (Fig. 4g, Additional file 1: Table S6). The H3K27 (H3) is antibody serving as positive control, and GAPDH loci were used as internal ChIP control for Ezh2 unbound region. The results revealed that Ezh2 directly binds to the PTEN promoter from 754 to 954 bp (Fig. 4h), suggesting that Ezh2 directly binds to the PTEN promoter to regulate its transcription.

\section{PTEN/Akt acts as the downstream component of Ezh2 and contributes to the effects of Ezh2 on the pluripotent and EMT phenotype in GC cells}

Finally, we conducted in vitro experiments to investigate whether Ezh2 functioned in a PTEN-mediated manner in GC. The enhanced or suppressed proliferative, invasion, sphere formation capacity in GC cells caused by overexpressing or knockdown Ezh2 was significantly attenuated or restored by upregulation or downregulation of PTEN (Fig. 5a-c). And protein expression levels of the EMT and pluripotent genes (E-cadherin, Vimentin, and Sox2 were both in consistent with the cellular biological behavior (Fig. 5d).

We further tested the change of AKT signaling and found that Ezh2 overexpression can induce AKT phosphorylation, while simultaneous overexpression of PTEN can suppress AKT phosphorylation (Fig. 5d). Similarly, when Ezh2 is silenced, pAKT is downregulated, while knockdown of PTEN at the same time can restore the expression of pAKT (Fig. 5d). These data indicated that upregulation of Ezh2 activates the AKT/PTEN pathway in GC cells. Our results indicated that Ezh2 induce the EMT and pluripotent phenotype of GC cell in a AKT/ PTEN-mediated manner.

\section{Discussion}

In this study, we determined the pivotal role of Ezh2 in GC pathogenesis and its underlying mechanisms. We observed that elevated Ezh2 expression was directly correlated with GC tumorigenesis and progression. High expression of Ezh2 could be used to identify a greatly increased risk of recurrence or invasion in patients after surgery, which might serve as a valuable prognostic marker. We also found that in addition to facilitating the proliferation and invasion of GC cells, Ezh2 especially lead to the EMT and pluripotent phenotype of GC cells through mediating the Akt/PTEN signaling pathway. Collectively, our clinical and mechanistic evidence strongly suggested that dysregulated Ezh2 expression mediating abnormal Akt/PTEN signaling critically contributes to GC pathogenesis and aggressive behavior.

The present study demonstrated that Ezh2 was overexpressed in GC specimens. Similar results have been obtained by other investigations, although the number of tumors analyzed was different and those studies focused on only the protein level $[30,31]$. These findings provide support for a role of elevated Ezh2 protein expression in the tumorigenesis of GC. More importantly, we demonstrate for the first time the potential role of deregulated Ezh2 mRNA in promoting GC progression and 
A

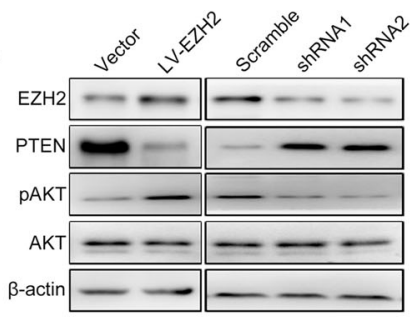

C

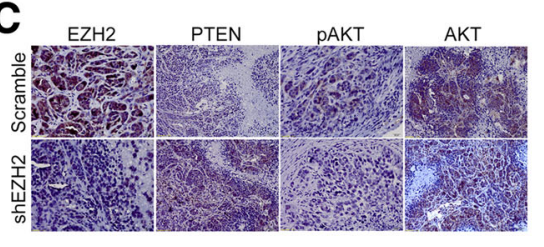

B

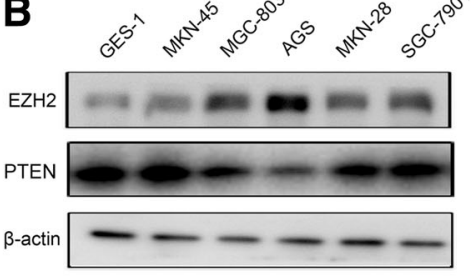

D

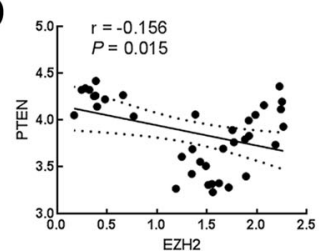

E
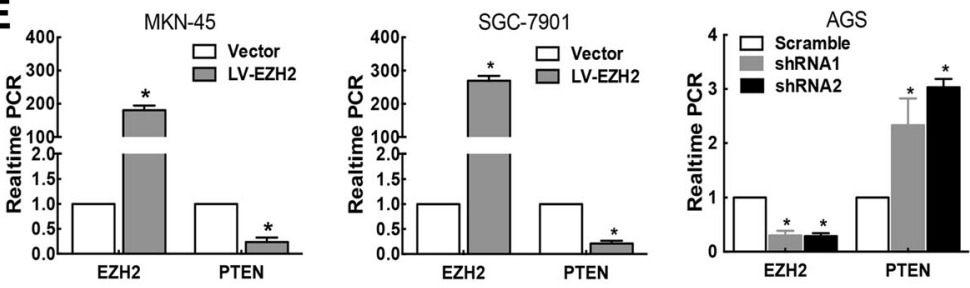

$\mathbf{F}$
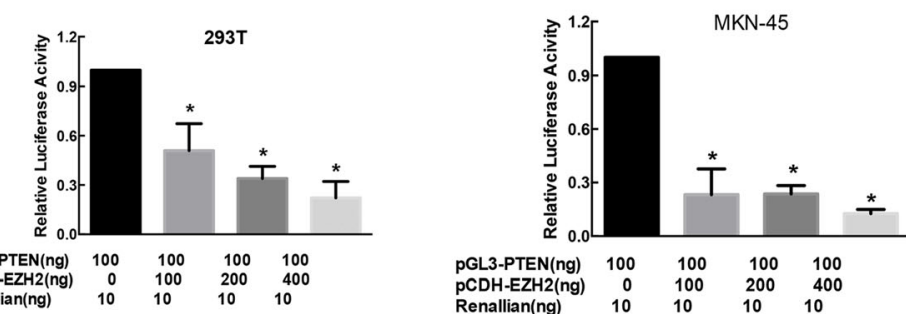

G

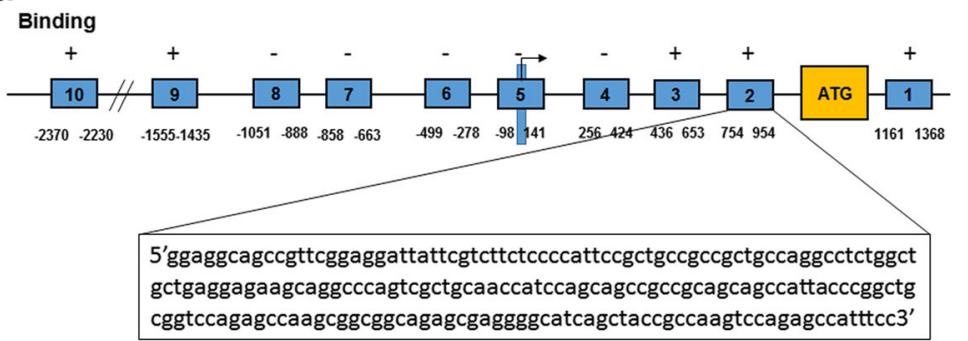

H
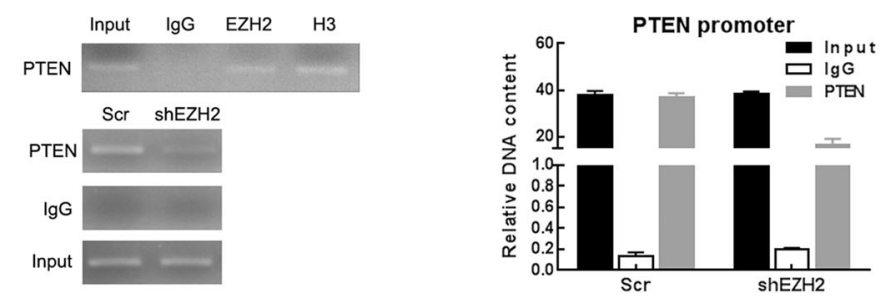

Fig. 4 (See legend on next page.) 
(See figure on previous page.)

Fig. 4 Ezh2 regulates PTEN/AKT signaling by directly binding to the promoter regions of PTEN in GC. a Representative images of the Western blot analysis for expression of Ezh2, PTEN, p-Akt, and total Akt in Ezh2-overexpressing MKN-45 and SGC-7901 cells and normal control, as well as Ezh2-knockdown AGS cells and normal control. b Representative images of the Western blot analysis for basic expression of Ezh2 and PTEN in five GC cell lines and the normal human gastric mucous cell line (GES-1). c Representative images of the IHC analysis for expression of Ezh2, PTEN, p-Akt, and total Akt in xenograft tissues. $\mathbf{d}$ Ezh2 and PTEN mRNA expression correlation analyses using the TCGA gastric cancer data. e The qRT-PCR results showed that PTEN mRNA was decreased in Ezh2-overexpressing MKN-45 and SGC-7901 cells, while increased in Ezh2-knockdown AGS cells. Data are represented as mean \pm SEM. ${ }^{*} P<0.01$. f Dual-reporter luciferase assays showed that overexpression of Ezh2 in HEK-293T and MKN-45 cells suppressed the promoter activity of PTEN. Data are represented as mean \pm SEM. ${ }^{*} P<0.05$. g Represent schemata of the PTEN promoter regions with or without binding affinity for EZH2. Arrow indicates the transcriptional start site. ATG indicates translation start codon. $\mathbf{h}$ ChIP assays showed that endogenous Ezh2 bound to the promoter region of PTEN. IgG served as a negative control, and H3K27 (H3) served as a positive control

unfavorable prognosis. We demonstrated that Ezh2 mRNA expression is significantly correlated with tumor stage and invasion. Further analysis demonstrated that Ezh2 mRNA, while not EZH2 protein expression, was an independent prognostic indicator for GC survival, strongly suggesting that GC patients with high Ezh2 expression should undergo follow-up at shorter intervals and be kept under close surveillance.

Our studies demonstrated that the toggle points protein of Akt signaling, PTEN, is transcriptionally regulated by Ezh2 in GC partly and contributes to the highproliferation and invasiveness activity, EMT, and pluripotent phenotype. It was previously identified that PTEN/ Akt signaling [32] and Ezh2 [33] are important regulators for the proliferation and invasion of gastric cancer cells. Here, we present a mechanism in consistent with these previous observations. Previous studies established the critical roles of PTEN/Akt signaling activity in tumor cell pluripotent phenotype [34]. The present study is the first to demonstrate that PTEN/Akt signaling activity is associated with Ezh2 activation in human GC, suggesting a link between PTEN/Akt signaling inactivation and Ezh2 activation. By using well-established Ezh2 upregulation and downregulation systems, we were able to show that aberrant expression of Ezh2 significantly impacted PTEN expression and Akt phosphorylation in GC cells. We also found that Ezh2 expression is involved in PTEN promoter activity in GC cells, and the binding is functional, which we confirmed by using luciferase and ChIP assay and rescue experiments. However, Ezh2 is a histone methyltransferase and the catalytic subunit of the Polycomb Repressive Complex 2 (PRC2), but not a transcription factor that binds a specific DNA sequence/motif. Thus, there should be other factors that help Ezh2 to bind to PTEN promoter. It has been found that imatinib could cause drug resistance via recruitment of EZH2 to the promoter region of the PTEN and then downregulates PTEN's transcripts in leukemia patients [35]; moreover, cumulative evidences revealed that lncRNAs interacted with EZH2 and recruited it to genes' promoter regions to repress genes' expression [36-38]. Furthermore, Chen NM and colleagues found that KRAS signaling was required for EZH2-mediated transcriptional activation of the inflammatory transcription factor nuclear factor of activated T cells 1 (NFATC1) in pancreatic ductal adenocarcinoma cells [39]. All these suggest that drugs, IncRNA, and transcription factor, may contribute to the binding of EZH2 to PTEN promoter. Further studies may focus on the exactly molecular contribution to EZH2 recruitment to the promoter region of PTEN.

Besides its aforementioned role, the implication of a role for Ezh2 in GC tumorigenesis and development is supported by lines of evidence suggesting that Ezh2 is essential for cellular EMT in GC [40-42]. In fact, Ezh2 regulates the expression of various EMT-related genes in various cancer types [40-42], suggesting that Ezh2 is required for EMT during tumor progression. In addition to the present study is the first to provide evidence of a critical role for activated Ezh2 in gastric cancer pluripotent phenotype by regulating PTEN/Akt signaling; it also shows a tight correlation between Ezh2 expression and E-cadherin and Vimentin expression mediate by PTEN/Akt signaling, supporting a critical role for Ezh2-PTEN/Akt axis in cellular EMT. Given the identified pivotal roles of Ezh2 in these two critical aspects of cancer biology, promotion of GC progression by activation of Ezh2 is conceivable.

Aberrant Ezh2 expression occurs in many other solid tumors, but its effects and underlying mechanism in these cancers are currently unclear. Whether Ezh2 is involved in the oncogenesis and development of other human malignancies via the PTEN/Akt signaling is still worth exploring, and the results would help us to better understand the role of Ezh2 in cancer.

\section{Conclusion}

In summary, we demonstrated that Ezh2 is overexpressed in primary GC and its expression is correlated with the tumor burden and clinical outcome. In addition, besides promoting GC cell proliferation and invasion, we identified Ezh2 as a key regulator of GC cell EMT and pluripotent phenotype via activation of the PTEN/Akt pathway. Our data strongly suggested that Ezh2 can be used for prognostication for GC and it may be utilized as a therapeutic target. Further exploration is necessary for the mechanism underlying Ezh2 overexpression in GC, particularly the molecule(s) to which it 

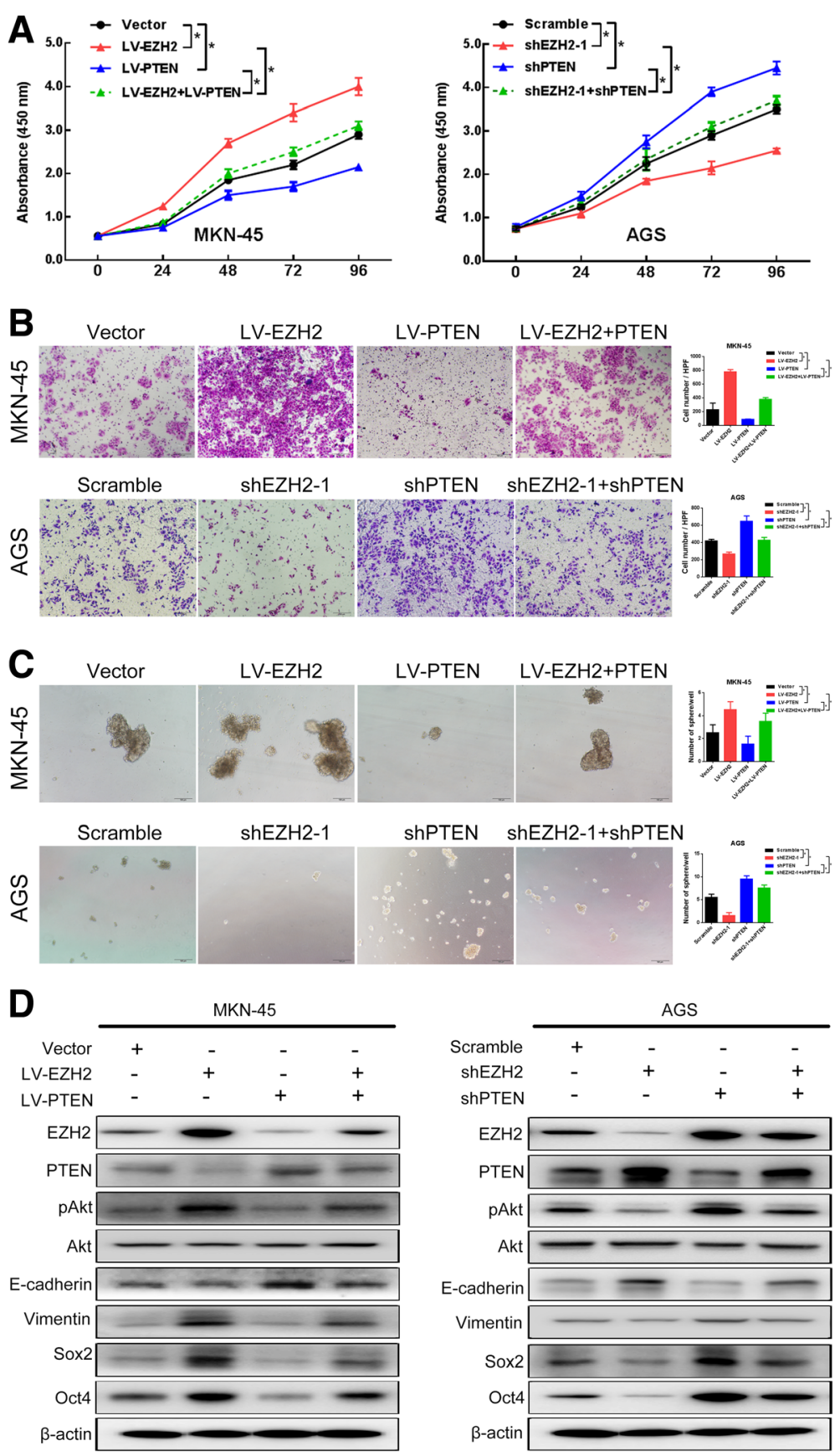

Fig. 5 Ezh2 facilitated GC progression in part via PTEN/Akt pathway. a The CCK-8 results showed that overexpression of PTEN partially attenuated the enhanced cell proliferation induced by overexpression of Ezh2 in MKN-45 cells, while knockdown of PTEN partially rescued the decreased cell proliferation induced by knockdown of Ezh2 in MKN-45 cells. Data are represented as mean \pm SEM. ${ }^{*} P<0.01$. b Representative images (left) and the number of invading cells (right) per high-power field showed that upregulation of PTEN partially attenuated the enhanced invasiveness ability of MKN-45 cells promoted by Ezh2 overexpression, while downregulation of PTEN partially rescued the decreased cell invasiveness of AGC cells promoted by Ezh2 knockdown. Data are represented as mean \pm SEM. ${ }^{*} P<0.01$. $\mathbf{c}$ Representative images (left) and the number of sphere formation (right) per high-power field showed that upregulation of PTEN partially attenuated the enhanced sphere formation of MKN-45 cells promoted by Ezh2 overexpression, while downregulation of PTEN partially rescued the decreased sphere formation activity of AGS cells promoted by Ezh2 knockdown. Data are represented as mean \pm SEM. ${ }^{*} P<0.01$. d Ezh2-overexpressing MKN-45 cells were transfected with empty vector or LV-PTEN, while Ezh2-knockdown AGS cells were transfected with scrambled or PTEN siRNA, and cells were subjected to western blotting with the indicated antibodies 
binds when modulating tumor cell behavior. Therefore, Ezh2 provides new perspectives for research of cell regulation in gastric cancer and new targets for gastric cancer diagnosis and treatment.

\section{Additional file}

Additional file 1: Materials and Methods. Table S1. Relationship between EZH2 expression and clinicopathologic parameters of gastric cancer patients. Table S2. Univariate and multivariate analysis of clinicopathological factors for disease-free survival in gastric cancer (qRT-PCR cohort). Table S3. Univariate and multivariate analysis of clinicopathological factors for overall survival in gastric cancer (qRT-PCR cohort). Table S4. Univariate and multivariate analysis of clinicopathological factors for overall survival in gastric cancer (IHC cohort). Table S5. Correlation analysis of expression of stem cell related factors with EZH2. Table S6. Primers and siRNA sequences used in this study. (DOCX $67 \mathrm{~kb})$

\section{Acknowledgements}

Not applicable.

\section{Funding}

This study was supported by National Natural Science Foundation of China (81602078), Hospital Foundation of Fudan University Shanghai Cancer Center (YJ201504), National Human Genetic Resources Sharing Service Platform (2005DKA21300), and Shanghai Natural Science Foundation (17ZR1406500). The roles of the funding body involved in the design of the study and collection, analysis, and interpretation of data and in writing the manuscript.

\section{Availability of data and materials}

Please contact the author for data requests.

\section{Authors' contributions}

L G, MD X, and RX H carried out the molecular genetic studies, participated in the sequence alignment, and drafted the manuscript. RX H and CT carried out the immunoassays. L G participated in the sequence alignment. WJ G and $M D X$ participated in the design of the study and performed the statistical analysis. WW W, JY Z, YW G, and ZH W conceived of the study, participated in its design and coordination, and helped to draft the manuscript. All authors read and approved the final manuscript.

\section{Ethics approval and consent to participate}

Ethics approval and consent to participate for the study was obtained from the Clinical Research Ethics Committee of Fudan University Shanghai Cancer Center (FUSCC), Ethical code: 050432-4-1212B.

\section{Consent for publication}

Consent to publish has been obtained from the participant.

\section{Competing interests}

The authors declare that they have no competing interests.

\section{Publisher's Note}

Springer Nature remains neutral with regard to jurisdictional claims in published maps and institutional affiliations.

\section{Author details}

'Department of Medical Oncology, Fudan University Shanghai Cancer Center, Shanghai 200032, China. ${ }^{2}$ Department of Oncology, Shanghai Medical College, Fudan University, Shanghai 200032, China. ${ }^{3}$ Department of Medical Oncology, Zhongshan Hospital, Fudan University, Shanghai 200032, China. ${ }^{4}$ Department of Pathology and tissue bank, Fudan University Shanghai Cancer Center, Shanghai 200032, China. ${ }^{5}$ Department of Oncology, The First Affiliated Hospital of Sun Yat-Sen University, Guangzhou, Guangdong 510000, China. ${ }^{6}$ Department of Pathology, Fudan University Shanghai Cancer Center, Shanghai 200032, China.
Received: 30 March 2017 Accepted: 26 December 2017

Published online: 15 January 2018

\section{References}

1. Siegel RL, Miller KD, Jemal A. Cancer statistics, 2016. CA Cancer J Clin. 2016; 66(1):7-30.

2. Park JY, von Karsa L, Herrero R. Prevention strategies for gastric cancer: a global perspective. Clinical endoscopy. 2014;47(6):478-89.

3. Chen $W$, Zheng $R$, Baade PD, Zhang S, Zeng H, Bray F, Jemal A, XQ Y, He J. Cancer statistics in China, 2015. CA Cancer J Clin. 2016;

4. Elimova E, Shiozaki H, Wadhwa R, Sudo K, Chen Q, Estrella JS, Blum MA, Badgwell B, Das P, Song S, et al. Medical management of gastric cancer: a 2014 update. World J Gastroenterol. 2014;20(38):13637-47.

5. Aoto T, Saitoh N, Sakamoto Y, Watanabe S, Nakao M. Polycomb group protein-associated chromatin is reproduced in post-mitotic G1 phase and is required for S phase progression. J Biol Chem. 2008;283(27):18905-15.

6. Zeidler M, Kleer CG. The Polycomb group protein Enhancer of Zeste 2: its links to DNA repair and breast cancer. J Mol Histol. 2006;37(5-7):219-23.

7. Liu TP, Lo HL, Wei LS, Hsiao HH, Yang PM. S-Adenosyl-L-methioninecompetitive inhibitors of the histone methyltransferase EZH2 induce autophagy and enhance drug sensitivity in cancer cells. Anti-Cancer Drugs. 2015:26(2):139-47.

8. Leseva M, Santostefano KE, Rosenbluth AL, Hamazaki T, Terada N. E2f6mediated repression of the meiotic Stag3 and Smc1beta genes during early embryonic development requires Ezh2 and not the de novo methyltransferase Dnmt3b. Epigenetics. 2013;8(8):873-84.

9. Kondo Y. Targeting histone methyltransferase EZH2 as cancer treatment. J Biochem. 2014;156(5):249-57.

10. Benard A, Goossens-Beumer IJ, van Hoesel AQ, Horati H, Putter H, Zeestraten EC, van de Velde CJ, Kuppen PJ. Prognostic value of polycomb proteins EZH2, BMI1 and SUZ12 and histone modification H3K27me3 in colorectal cancer. PLoS One. 2014;9(9):e108265.

11. Gao SB, Zheng QF, Xu B, Pan CB, Li KL, Zhao Y, Zheng QL, Lin X, Xue LX, Jin $\mathrm{GH}$. EZH2 represses target genes through H3K27-dependent and H3K27independent mechanisms in hepatocellular carcinoma. Mol Cancer Res. 2014;12(10):1388-97

12. Geng J, Li X, Zhou Z, CL W, Dai M, Bai X. EZH2 promotes tumor progression via regulating VEGF-A/AKT signaling in non-small cell lung cancer. Cancer Lett. 2015;359(2):275-87.

13. Li Z, Xu L, Tang N, Xu Y, Ye X, Shen S, Niu X, Lu S, Chen Z. The polycomb group protein $E Z H 2$ inhibits lung cancer cell growth by repressing the transcription factor Nrf2. FEBS Lett. 2014;588(17):3000-7.

14. Cai GH, Wang K, Miao Q, Peng YS, Chen XY. Expression of polycomb protein EZH2 in multi-stage tissues of gastric carcinogenesis. J Dig Dis. 2010;11(2):88-93.

15. Choi JH, Song YS, Yoon JS, Song KW, Lee YY. Enhancer of zeste homolog 2 expression is associated with tumor cell proliferation and metastasis in gastric cancer. APMIS. 2010;118(3):196-202.

16. Matsukawa $Y$, Semba S, Kato H, Ito A, Yanagihara K, Yokozaki H. Expression of the enhancer of zeste homolog 2 is correlated with poor prognosis in human gastric cancer. Cancer Sci. 2006:97(6):484-91.

17. Liu YW, Sun M, Xia R, Zhang EB, Liu XH, Zhang ZH, Xu TP, De W, Liu BR, Wang ZX. LincHOTAIR epigenetically silences miR34a by binding to PRC2 to promote the epithelial-to-mesenchymal transition in human gastric cancer. Cell Death Dis. 2015:6:e1802.

18. Wang YJ, Liu JZ, Lv P, Dang Y, Gao JY, Wang Y. Long non-coding RNA CCAT2 promotes gastric cancer proliferation and invasion by regulating the E-cadherin and LATS2. Am J Cancer Res. 2016;6(11):2651-60.

19. Cheng LL, Itahana Y, Lei ZD, Chia NY, Wu Y, Yu Y, Zhang SL, Thike AA, Pandey A, Rozen S, et al. TP53 genomic status regulates sensitivity of gastric cancer cells to the histone methylation inhibitor 3-deazaneplanocin A (DZNep). Clin Cancer Res. 2012;18(15):4201-12

20. Lauwers GY CF, Graham DY, Curado MP Tumours of the stomach. WHO classification of tumours of the digestive system Bosman FT CF, Hruban RH, Theise ND; 2010: 48-59.

21. Du X, MD X, Wang Y, Weng WW, Wei P, Qi P, Zhang Q, Tan C, Ni S, Dong L, et al. A positive feedback loop of IncRNA-PVT1 and FOXM1 facilitates gastric cancer growth and invasion. Clin Cancer Res. 2016;

22. Qi P, MD X, Shen XH, Ni SJ, Huang D, Tan C, Weng WW, Sheng WQ, Zhou $X Y$, Du X. Reciprocal repression between TUSC7 and miR-23b in gastric cancer. International journal of cancer Journal international du cancer. 2015; 
23. Gan L, He J, Zhang X, Zhang YJ, GZ Y, Chen Y, Pan J, Wang JJ, Wang X. Expression profile and prognostic role of sex hormone receptors in gastric cancer. BMC Cancer. 2012;12:566.

24. Nie W, MD X, Gan L, Huang H, Xiu Q, Li B. Overexpression of stathmin 1 is a poor prognostic biomarker in non-small cell lung cancer. Lab Investig. 2015; 95(1):56-64.

25. Liu N, Chen NY, Cui RX, Li WF, Li Y, Wei RR, Zhang MY, Sun Y, Huang BJ, Chen M, et al. Prognostic value of a microRNA signature in nasopharyngeal carcinoma: a microRNA expression analysis. The Lancet Oncology. 2012;13(6):633-41.

26. Yan W, Fu Y, Tian D, Liao J, Liu M, Wang B, Xia L, Zhu Q, Luo M. PI3 kinase/Akt signaling mediates epithelial-mesenchymal transition in hypoxic hepatocellular carcinoma cells. Biochem Biophys Res Commun. 2009;382(3):631-6.

27. Meadows KN, lyer S, Stevens MV, Wang D, Shechter S, Perruzzi C, Camenisch TD, Benjamin LE. Akt promotes endocardial-mesenchyme transition. J Angiogenes Res. 2009;1:2.

28. Gargini R, Cerliani JP, Escoll M, Anton IM, Wandosell F. Cancer stem cell-like phenotype and survival are coordinately regulated by Akt/FoxO/Bim pathway. Stem cells (Dayton, Ohio). 2015;33(3):646-60.

29. Li B, Lu Y, Wang H, Han X, Mao J, Li J, Yu L, Wang B, Fan S, Yu X, et al. miR221/222 enhance the tumorigenicity of human breast cancer stem cells via modulation of PTEN/Akt pathway. Biomedicine \& pharmacotherapy $=$ Biomedecine \& pharmacotherapie. 2016;79:93-101.

30. Guo L, Yang TF, Liang SC, Guo JX, Wang Q. Role of EZH2 protein expression in gastric carcinogenesis among Asians: a meta-analysis. Tumour Biol. 2014; 35(7):6649-56.

31. Wang W, Wang F, Zong G, Liu R, Zhang Y, Luan Y, Xu L, Xuan J. Prognostic significance of EZH2 expression in patients with digestive cancers: a metaanalysis. Int J Clin Exp Med. 2015;8(9):16043-9.

32. Zhang L, Wu Z, Ma Z, Liu H, Wu Y, Zhang Q. WWP1 as a potential tumor oncogene regulates PTEN-Akt signaling pathway in human gastric carcinoma. Tumour Biol. 2015;36(2):787-98.

33. Sun M, Nie F, Wang Y, Zhang Z, Hou J, He D, Xie M, De W, Wang Z, Wang J. LncRNA HOXA11-AS promotes proliferation and invasion of gastric cancer by scaffolding the chromatin modification factors PRC2, LSD1 and DNMT1. Cancer Res. 2016;

34. Schubbert S, Jiao J, Ruscetti M, Nakashima J, Wu S, Lei H, Xu Q, Yi W, Zhu H, Wu H. Methods for PTEN in stem cells and cancer stem cells. Methods in molecular biology (Clifton, NJ). 2016;1388:233-85.

35. Nishioka C, Ikezoe T, Yang J, Udaka K, Yokoyama A. Imatinib causes epigenetic alterations of PTEN gene via upregulation of DNA methyltransferases and polycomb group proteins. Blood cancer journal. 2011;1(12):e48.

36. Chen JH, Zhou LY, Xu S, Zheng YL, Wan YF, Hu CP. Overexpression of IncRNA HOXA11-AS promotes cell epithelial-mesenchymal transition by repressing miR-200b in non-small cell lung cancer. Cancer Cell Int. 2017;17:64.

37. Liu Z, Chen Z, Fan R, Jiang B, Chen X, Chen Q, Nie F, Lu K, Sun M. Overexpressed long noncoding RNA HOXA11-AS promotes cell cycle progression and metastasis in gastric cancer. Mol Cancer. 2017;16(1):82.

38. Huang M, Hou J, Wang Y, Xie M, Wei C, Nie F, Wang Z, Sun M. Long noncoding RNA LINC00673 is activated by SP1 and exerts oncogenic properties by interacting with LSD1 and EZH2 in gastric cancer. Mol Ther. 2017;25(4):1014-26.

39. Chen NM, Neesse A, Dyck ML, Steuber B, Koenig AO, Lubeseder-Martellato C, Winter T, Forster T, Bohnenberger H, Kitz J, et al. Context-dependent epigenetic regulation of nuclear factor of activated T cells 1 in pancreatic plasticity. Gastroenterology. 2017:152(6):1507-1520.e1515.

40. Battistelli C, Cicchini C, Santangelo L, Tramontano A, Grassi L, Gonzalez FJ, de Nonno V, Grassi G, Amicone L, Tripodi M. The Snail repressor recruits EZH2 to specific genomic sites through the enrollment of the IncRNA HOTAIR in epithelial-to-mesenchymal transition. Oncogene. 2016;

41. Cardenas H, Zhao J, Vieth E, Nephew KP, Matei D. EZH2 inhibition promotes epithelial-to-mesenchymal transition in ovarian cancer cells. Oncotarget. 2016;

42. Chang JW, Gwak SY, Shim GA, Liu L, Lim YC, Kim JM, Jung MG, Koo BS. $\mathrm{EZH} 2$ is associated with poor prognosis in head-and-neck squamous cell carcinoma via regulating the epithelial-to-mesenchymal transition and chemosensitivity. Oral Oncol. 2016;52:66-74.

\section{Submit your next manuscript to BioMed Central and we will help you at every step:}

- We accept pre-submission inquiries

- Our selector tool helps you to find the most relevant journal

- We provide round the clock customer support

- Convenient online submission

- Thorough peer review

- Inclusion in PubMed and all major indexing services

- Maximum visibility for your research

Submit your manuscript at www.biomedcentral.com/submit

C) Biomed Central 